\title{
Front Matter: Volume 9083
}

, "Front Matter: Volume 9083," Proc. SPIE 9083, Micro- and Nanotechnology Sensors, Systems, and Applications VI, 908301 (25 June 2014); doi: 10.1117/12.2072119

SPIE. Event: SPIE Defense + Security, 2014, Baltimore, MD, United States 


\section{PROCEEDINGS OF SPIE}

\section{Micro- and Nanotechnology Sensors, Systems, and Applications VI}

Thomas George

M. Saif Islam

Achyut K. Dutta

Editors

5-9 May 2014

Baltimore, Maryland, United States

Sponsored and Published by

SPIE

Volume 9083 
The papers included in this volume were part of the technical conference cited on the cover and title page. Papers were selected and subject to review by the editors and conference program committee. Some conference presentations may not be available for publication. The papers published in these proceedings reflect the work and thoughts of the authors and are published herein as submitted. The publisher is not responsible for the validity of the information or for any outcomes resulting from reliance thereon.

Please use the following format to cite material from this book:

Author(s), "Title of Paper," in Micro- and Nanotechnology Sensors, Systems, and Applications VI, edited by Thomas George, M. Saif Islam, Achyut K. Dutta, Proceedings of SPIE Vol. 9083 (SPIE, Bellingham, WA, 2014) Article CID Number.

ISSN: 0277-786X

ISBN: 9781628410204

Published by

SPIE

P.O. Box 10, Bellingham, Washington 98227-0010 USA

Telephone +1 3606763290 (Pacific Time) · Fax +1 3606471445

SPIE.org

Copyright @ 2014, Society of Photo-Optical Instrumentation Engineers.

Copying of material in this book for internal or personal use, or for the internal or personal use of specific clients, beyond the fair use provisions granted by the U.S. Copyright Law is authorized by SPIE subject to payment of copying fees. The Transactional Reporting Service base fee for this volume is $\$ 18.00$ per article (or portion thereof), which should be paid directly to the Copyright Clearance Center (CCC), 222 Rosewood Drive, Danvers, MA 01923. Payment may also be made electronically through CCC Online at copyright.com. Other copying for republication, resale, advertising or promotion, or any form of systematic or multiple reproduction of any material in this book is prohibited except with permission in writing from the publisher. The CCC fee code is 0277-786X/14/\$18.00.

Printed in the United States of America.

Publication of record for individual papers is online in the SPIE Digital Library.

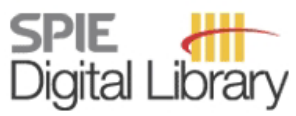

SPIEDigitalLibrary.org

Paper Numbering: Proceedings of SPIE follow an e-First publication model, with papers published first online and then in print and on CD-ROM. Papers are published as they are submitted and meet publication criteria. A unique, consistent, permanent citation identifier (CID) number is assigned to each article at the time of the first publication. Utilization of CIDs allows articles to be fully citable as soon as they are published online, and connects the same identifier to all online, print, and electronic versions of the publication. SPIE uses a six-digit CID article numbering system in which:

- The first four digits correspond to the SPIE volume number.

- The last two digits indicate publication order within the volume using a Base 36 numbering

system employing both numerals and letters. These two-number sets start with 00, 01, 02, 03, 04, $05,06,07,08,09,0 A, 0 B \ldots 0 Z$, followed by 10-1Z, 20-2Z, etc.

The CID Number appears on each page of the manuscript. The complete citation is used on the first page, and an abbreviated version on subsequent pages. Numbers in the index correspond to the last two digits of the six-digit CID Number. 


\section{Contents}

TWO-DIMENSIONAL NANO-LAYERED SYSTEMS: GRAPHENE AND BEYOND

908302 Two-dimensional atomic crystals beyond grapheme (Keynote Paper) [9083-1]

A. B. Kaul, National Science Foundation (United States)

908304 Novel layered two-dimensional semiconductors as the building blocks for nanoelectronic/photonic systems (Invited Paper) [9083-3]

G. Su, D. De, V. G. Hadjiev, H. Peng, Univ. of Houston (United States)

908305 Graphene and beyond-graphene 2D crystals for next-generation green electronics (Invited Paper) [9083-4]

J. Kang, W. Cao, X. Xie, D. Sarkar, W. Liu, K. Banerjee, Univ. of California, Santa Barbara (United States)

908307 High-field and thermal transport in 2D atomic layer devices (Invited Paper) [9083-6] A. Serov, V. E. Dorgan, A. Behnam, Univ. of Illinois at Urbana-Champaign (United States); C. D. English, Stanford Univ. (United States); Z. Li, Stanford Univ. (United States) and Univ. of Illinois at Urbana-Champaign (United States); S. Islam, Univ. of Illinois at Urbana-Champaign (United States); E. Pop, Stanford Univ. (United States) and Univ. of Illinois at Urbana-

Champaign (United States)

\section{EMERGING ELECTRONIC DEVICES/SYSTEMS BASED ON ADAPTIVE AND METAMATERIALS I}

908309 Coherent phonons in carbon based nanostructures (Invited Paper) [9083-8]

G. D. Sanders, Univ. of Florida (United States); A. R. T. Nugraha, K. Sato, Tohoku Univ. (Japan); J.-H. Kim, Sungkyunkwan Univ. (Korea, Republic of); Y.-S. Lim, Konkuk Univ. (Korea, Republic of); J. Kono, Rice Univ. (United States); R. Saito, Tohoku Univ. (Japan);

C. J. Stanton, Univ. of Florida (United States)

9083 OA Double graphene-layer structures for adaptive devices (Invited Paper) [9083-9]

V. Mitin, Univ. at Buffalo (United States); V. Ryzhii, T. Ołsuji, Tohoku Univ. (Japan); M. Ryzhii,

Univ. of Aizu (Japan); M. S. Shur, Rensselaer Polytechnic Institute (United States)

\section{EMERGING ELECTRONIC DEVICES/SYSTEMS BASED ON ADAPTIVE AND METAMATERIALS II}

9083 OB Terahertz devices and device modeling (Invited Paper) [9083-10]

R. Soligo, M. Saraniti, S. M. Goodnick, Arizona State Univ. (United States)

9083 OC Investigation of MEMS bi-material sensors with metamaterial absorbers for THz imaging (Invited Paper) [9083-11]

F. Alves, D. Grbovic, G. Karunasiri, Naval Postgraduate School (United States) 
9083 OD Growth of 2D heterostructures of graphene/BN (Invited Paper) [9083-12]

J. Hwang, B. R. Calderon, H. A. Alsalman, J. Y. Kwak, M. Kim, M. G. Spencer, Cornell Univ. (United States)

$9083 \mathrm{Ol} \quad$ Graphene based active and passive component development on transparent substrates (Invited Paper) [9083-17]

J. H. Schaffner, K. Son, H. J. Song, J. S. Moon, A. A. Kiselev, H.-C. Seo, B. Yang, D. Wong, HRL Labs., LLC (United States)

1D NANOELECTRONICS: NANOWIRE AND NANOTUBE ARCHITECTURE TRANSISTORS

9083 OK Group IV nanotube transistors for next generation ubiquitous computing (Invited Paper) [9083-19]

H. M. Fahad, A. M. Hussain, G. A. Sevilla Torres, King Abdullah Univ. of Science and Technology (Saudi Arabia); S. K. Banerjee, The Univ. of Texas at Austin (United States);

M. M. Hussain, King Abdullah Univ. of Science and Technology (Saudi Arabia)

9083 OM En route toward high performance electronics based on single-walled carbon nanotubes (Invited Paper) [9083-21]

Q. Cao, IBM Thomas J. Watson Research Ctr. (United States)

MICRONANO TECHNOLOGIES FOR ADAPTIVE OPTICS AND BEAM CONTROL

9083 ON Adaptive optics center of excellence for national security (Keynote Paper) [9083-22]

B. Agrawal, Naval Postgraduate School (United States)

$908300 \quad$ Advanced deformable mirrors for high-power lasers (Invited Paper) [9083-23]

J. Mansell, J. Jameson, B. Henderson, MZA Associates Corp. (United States)

$90830 Q \quad$ Beam control in multiphoton microscopy using a MEMS spatial light modulator (Invited Paper) [9083-25]

T. Bifano, H. Paudel, Boston Univ. (United States)

9083 OR Adaptive optics correction of a laser beam propagating underwater (Invited Paper) [9083-1 17]

S. R. Restaino, W. Hou, A. Kanaev, S. Matt, C. Font, U.S. Naval Research Lab. (United States)

MICRONANO SENSOR SYSTEMS FOR POWER AND CHEMICAL PRODUCTION APPLICATIONS

9083 ОT Laser-absorption sensing of gas composition of products from coal gasification (Invited Paper) [9083-28]

J. B. Jeffries, R. Sur, K. Sun, R. K. Hanson, Stanford Univ. (United States)

iv 
9083 OU Industrial Raman gas sensing for real-time system control (Invited Paper) [9083-29] M. Buric, J. Mullen, B. Chorpening, S. Woodruff, National Energy Technology Lab. (United States)

9083 OV Optical fiber Fabry-Perot interferometry (Invited Paper) [9083-30]

A. Wang, Virginia Polytechnic Institute and State Univ. (United States)

9083 OW Novel strategies for development of gas sensors for combustion and medical applications (Invited Paper) [9083-31]

A. Fulmer, M. Mullen, C. Sun, P. K. Dutta, The Ohio State Univ. (United States)

9083 OX Trace chemical detection in air using electronic noses and nano-structured sensing materials [9083-119]

K. D. Benkstein, C. B. Montgomery, S. Semancik, National Institute of Standards and Technology (United States)

9083 OY Metal oxide gas sensors on the nanoscale (Invited Paper) [9083-33]

A. Plecenik, Comenius Univ. in Bratislava (Slovakia); A. A. Haidry, Comenius Univ. in Bratislava (Slovakia) and German Aerospace Ctr. (Germany); T. Plecenik, P. Durina, M. Truchly, Comenius Univ. in Bratislava (Slovakia); M. Mosko, Comenius Univ. in Bratislava (Slovakia) and Institute of Electrical Engineering, SAS (Slovakia); B. Grancic, M. Gregor, T. Roch, L. Satrapinskyy, Comenius Univ. in Bratislava (Slovakia); A. Moskova, Institute of Electrical Engineering, SAS (Slovakia); M. Mikula, P. Kus, Comenius Univ. in Bratislava (Slovakia)

$90830 Z$ Use of nanostructured oxides for selective gas-sensing (Invited Paper) [9083-34] Y. Gönüllü, German Aerospace Ctr. (Germany) and Univ. of Cologne (Germany); B. Saruhan, German Aerospace Ctr. (Germany)

$908310 \quad$ Novel sensors to enable closed-loop active clearance control in gas turbine engines (Invited Paper) [9083-35]

J. Geisheimer, T. Holst, Meggitt Sensing Systems (United States)

ADVANCED MICRO/NANOTECHNOLOGIES FOR SOLAR ENERGY GENERATION: JOINT SESSION WITH CONFERENCES 9083 AND 9115

908312 Fundamentals and recent results of super high-efficiency solar cells (Invited Paper) [9083-38]

M. Yamaguchi, K. Ikeda, Toyota Technological Institute (Japan); T. Takamoto, Sharp Corp. (Japan); N. Kojima, Y. Ohshita, Toyota Technological Institute (Japan)

908313 Nanoscale optimization of quantum dot media for effective photovoltaic conversion (Invited Paper) [9083-39]

K. A. Sablon, U.S. Army Research Lab. (United States); A. Sergeev, Univ. at Buffalo (United States); J. W. Little, U.S. Army Research Lab. (United States); N. Vagidov, V. Mitin, Univ. at Buffalo (United States) 
908315 Optical modular arithmetic (Invited Paper) [9083-41]

D. S. Pavlichin, H. Mabuchi, Stanford Univ. (United States)

908317 Traveling-wave photon-phonon coupling as the basis for new signal processing technologies (Invited Paper) [9083-43]

P. T. Rakich, Yale Univ. (United States); J. A. Cox, Sandia National Labs. (United States);

H. Shin, Yale Univ. (United States); Z. Wang, H. Mousavi, H. Dong, The Univ. of Texas at Austin (United States); R. Jarecki, A. Siddiqui, Sandia National Labs. (United States); R. C. Potter, Rockwell Collins, Inc. (United States)

\section{D PRINTING: AN EMERGING TECHNOLOGY FOR MICRO/NANO DEVICE FABRICATION}

9083 ID 3-D printing of liquid metals for stretchable and flexible conductors (Invited Paper) [9083-49]

C. Trlica, D. P. Parekh, L. Panich, C. Ladd, M. D. Dickey, North Carolina State Univ. (United States)

\section{FLEXIBLE ELECTRONICS: MULTIFACETED EVOLUTIONS AND APPLICATIONS}

9083 IF Implementing MEMS technology for soft, (bio)electronics interfaces (Invited Paper) [9083-51]

A. Romeo, Y. Hofmeister, S. P. Lacour, Ecole Polytechnique Fédérale de Lausanne (Switzerland)

9083 1G Advanced flexible electronics: challenges and opportunities (Invited Paper) [9083-52] S. W. Bedell, D. Shahrjerdi, K. Fogel, P. Lauro, C. Bayram, B. Hekmatshoar, N. Li, J. Ott, D. Sadana, IBM Thomas J. Watson Research Ctr. (United States)

9083 1H High performance bio-integrated devices (Invited Paper) [9083-53] D.-H. Kim, J. Lee, M. Park, Seoul National Univ. (Korea, Republic of)

$908311 \quad H i g h-p e r f o r m a n c e$ flexible microwave passives on plastic (Invited Paper) [9083-57] Z. Ma, J.-H. Seo, S. J. Cho, Univ. of Wisconsin-Madison (United States); W. Zhou, The Univ. of Texas at Arlington (United States)

$90831 \mathrm{~J}$ Mechanics of flexible electronics and photonics based on inorganic micro- and nanomaterials (Invited Paper) [9083-55]

N. Lu, S. Yang, S. Qiao, The Univ. of Texas at Austin (United States)

$90831 \mathrm{~K}$ Transformational electronics: a powerful way to revolutionize our information world (Invited Paper) [9083-54]

J. P. Rojas, G. A. Torres Sevilla, M. T. Ghoneim, A. M. Hussain, S. M. Ahmed, J. M. Nassar, R. R. Bahabry, M. Nour, A. T. Kutbee, E. Byas, B. Al-Saif, A. M. Alamri, M. M. Hussain, King Abdullah Univ. of Science and Technology (Saudi Arabia) 
9083 1L Arthropod eye-inspired digital camera with unique imaging characteristics (Invited Paper) [9083-58]

J. Xiao, Univ. of Colorado Boulder (United States); Y. M. Song, Y. Xie, V. Malyarchuk, Univ. of Illinois at Urbana-Champaign (United States); I. Jung, Kyung Hee Univ. (Korea, Republic of); K.-J. Choi, Univ. of Illinois at Urbana-Champaign (United States); Z. Liu, A*STAR Institute of High Performance Computing (Singapore); H. Park, Harvard Univ. (United States); C. Lu, Zhejiang Univ. (China) and Northwestern Univ. (United States); R.-H. Kim, Univ. of Illinois at Urbana-Champaign (United States); R. Li, Northwestern Univ. (United States) and Dalian Univ. of Technology (China); K. B. Crozier, Harvard Univ. (United States); Y. Huang, Northwestern Univ. (United States); J. A. Rogers, Univ. of Illinois at Urbana-Champaign (United States)

$90831 \mathrm{M}$ Soft bio-integrated systems for continuous health monitoring (Invited Paper) [9083-59] M. Raj, P. H. Wei, B. Morey, X. Wang, B. Keen, P. DePetrillo, Y. Y. Hsu, R. Ghaffari, MC10, Inc. (United States)

9083 iN Two-dimensional atomic sheets for heterogeneous flexible high-frequency and low-power nanoelectronics (Invited Paper) [9083-56]

D. Akinwande, The Univ. of Texas at Austin (United States)

MICRO AUTONOMOUS SYSTEMS TECHNOLOGY (MAST): PERFORMANCE BOUNDS AND TRADE SPACE STUDIES: JOINT SESSION WITH CONFERENCES 9083/9084/9096

$908310 \quad$ From wakes to wings: using a multi-fidelity approach to design flapping wings (Invited Paper) [9083-60]

D. J. Willis, Univ. of Massachusetts Lowell (United States); H. Salehipour, Univ. of Toronto (Canada)

9083 IP Characterization and enhancement of micro brushless DC motor response (Invited Paper) [9083-61]

J. Conroy, U.S. Army Research Lab. (United States); A. Kehlenbeck, J. S. Humbert, Univ. of Maryland, College Park (United States); W. Nothwang, U.S. Army Research Lab. (United States)

$90831 Q \quad$ Power and weight considerations in small, agile quadrotors (Invited Paper) [9083-62]

Y. Mulgaonkar, M. Whitzer, Univ. of Pennsylvania (United States); B. Morgan,

C. M. Kroninger, A. M. Harrington, U.S. Army Research Lab. (United States); V. Kumar, Univ. of Pennsylvania (United States)

9083 IR Endurance bounds of aerial systems (Invited Paper) [9083-63]

A. M. Harrington, C. M. Kroninger, U.S. Army Research Lab. (United States)

9083 is Autonomous charging to enable long-endurance missions for small aerial robots (Invited Paper) [9083-64]

Y. Mulgaonkar, V. Kumar, Univ. of Pennsylvania (United States) 
MICRO AUTONOMOUS SYSTEMS TECHNOLOGY (MAST): POWER SOLUTIONS: JOINT SESSION WITH CONFERENCES $9083 / 9084 / 9096$

9083 IT MEMS-based and switched-capacitor approaches for miniature power supply applications (Invited Paper) [9083-65]

S. S. Bedair, C. D. Meyer, N. Lazarus, U.S. Army Research Lab. (United States); C. Dougherty, Univ. of Florida (United States); J. S. Pulskamp, B. Morgan, R. Polcawich, U.S. Army Research Lab. (United States); X. Lin, R. Bashirullah, Univ. of Florida (United States); I. Kierzewski, J. Martin, B. Power, U.S. Army Research Lab. (United States)

$90831 \mathrm{U}$ Power management for small scale systems (Invited Paper) [9083-66]

C. D. Meyer, S. S. Bedair, B. C. Morgan, U.S. Army Research Lab. (United States); X. Lin, R. Bashirullah, D. P. Arnold, Univ. of Florida (United States); I. M. Kierzewski, N. S. Lazarus, U.S. Army Research Lab. (United States)

9083 IV High specific energy and specific power aluminum/air battery for micro air vehicles (Invited Paper) [9083-67]

A. Kindler, L. Matthies, Jet Propulsion Lab. (United States)

9083 IW Thermophotovoltaic and thermoelectric portable power generators (Invited Paper) [9083-68]

W. R. Chan, Massachusetts Institute of Technology (United States); C. M. Waits, U.S. Army Research Lab. (United States); J. D. Joannopoulos, I. Celanovic, Massachusetts Institute of Technology (United States)

SELF-ASSEMBLED, BLOCK-COPOLYMER, NANO-STRUCTURES FOR ENERGY AND SENSOR APPLICATIONS

$90831 \mathrm{X}$ Self-assembled nanostructures as templates for patterned surfaces with nonmicroelectronic applications (Keynote Paper) [9083-69] J. Kerry, Univ. College Cork (Ireland); T. Ghoshal, M. A. Morris, Univ. College Cork (Ireland) and Trinity College Dublin (Ireland)

9083 IY Block copolymer self assembly for design and vapor phase synthesis of one-dimensional nanostructured materials (Invited Paper) [9083-70]

A. Rahman, C. T. Black, Brookhaven National Lab. (United States)

908320 Soft matter design principles for inorganic photonic nanoarchitectures in photovoltaics, colorimetric sensing, and self-cleaning antireflective coatings (Invited Paper) [9083-72] S. Guldin, Ecole Polytechnique Fédérale de Lausanne (Switzerland); U. Steiner, Univ. of Cambridge (United Kingdom) 
INNOVATIONS IN MULTIMODAL MOLECULAR PROBES: JOINT SESSION WITH CONFERENCES 9083 AND 9107

908325 Quantitative simultaneous PET-MR imaging (Invited Paper) [9083-77]

J. Ouyang, Massachusetts General Hospital (United States) and Harvard Medical School (United States); Y. Petibon, Massachusetts General Hospital (United States) and Lab. d'Imagerie Biomédicale, CNRS, Univ. Pierre et Marie Curie Paris 6 (France); C. Huang, Massachusetts General Hospital (United States) and Harvard Medical School (United States); T. G. Reese, Harvard Medical School (United States) and Martinos Ctr. for Biomedical Imaging (United States); A. L. Kolnick, Massachusetts General Hospital (United States); G. El Fakhri, Massachusetts General Hospital (United States) and Harvard Medical School (United States)

908326 The new frontiers of multimodality and multi-isotope imaging (Invited Paper) [9083-78] B. Behnam Azad, S. Nimmagadda, Johns Hopkins Univ. (United States)

HIGH-ACCURACY SPACE-BASED RADIOMETRY

908329 NASA ESTO's strategic investments in space-based radiometer technology and flight validation (Keynote Paper) [9083-81]

C. D. Norton, Jet Propulsion Lab. (United States) and NASA Goddard Space Flight Ctr. (United States); M. P. Pasciuto, NASA Goddard Space Flight Ctr. (United States)

9083 2A The power of inexpensive satellite constellations (Invited Paper) [9083-82]

L. P. Dyrud, R. La Tour, Draper Lab. (United States); W. H. Swartz, The Johns Hopkins Univ. Applied Physics Lab. LLC (United States); S. Nag, Massachusetts Institute of Technology (United States); S. R. Lorentz, L-1 Standards and Technology, Inc. (United States); T. Hilker, Oregon State Univ. (United States); W. J. Wiscombe, NASA Goddard Space Flight Ctr. (United States); S. J. Papadakis, The Johns Hopkins Univ. Applied Physics Lab. LLC (United States)

9083 2D Photon filter for energetic neutral atom detectors from carbon nanotubes (Invited Paper) [9083-85]

D. M. Deglau, D. G. Mitchell, A. H. Monica, B. Andrews, J. E. Mattson, S. J. Papadakis, The Johns Hopkins Univ. Applied Physics Lab. LLC (United States)

NANOTECHNOLOGY FOR MILLIMETER-WAVE SENSING II: JOINT SESSION WITH CONFERENCES 9083 AND 9078

$90832 \mathrm{G}$ Case study of concealed weapons detection at stand-off distances using a compact, large field-of-view THz camera (Invited Paper) [9083-89]

L. E. Marchese, M. Terroux, D. Dufour, M. Bolduc, C. Chevalier, F. Généreux, H. Jerominek, A. Bergeron, INO (Canada) 
MICRO/NANOTECHNOLOGIES FOR LASERS AND STANDOFF DETECTION I: JOINT SESSION WITH CONFERENCES 9083/9073/9106

$908321 \quad$ Ultrafast bandgap technique: light-induced semiconductor augmentation (Invited Paper) [9083-91]

I. K. Zakharova, M. K. Rafailov, Univ. of Alberta (Canada)

MICRO/NANOTECHNOLOGIES FOR LASERS AND STANDOFF DETECTION II: JOINT SESSION WITH CONFERENCES $9083 / 9073 / 9106$

$90832 \mathrm{~N} \quad$ Point and standoff detection of trace explosives using quantum cascade lasers (Invited Paper) [9083-96]

S. Kim, Univ. of Calgary (Canada); D. Lee, X. Liu, C. W. Van Neste, T. Thundat, Univ. of Alberta (Canada)

POSTER SESSION

9083 2P Digital level micro-fabrication for advanced optical structures [9083-100]

D. J. Boudreau, M. Christophersen, M. K. Yetzbacher, U.S. Naval Research Lab. (United States); M. J. DePrenger, Tekla Research Inc. (United States)

$90832 \mathrm{Q}$ Wavelength-selective visible-light detector based on integrated graphene transistor and surface plasmon coupler [9083-102]

C. W. Smith, D. Maukonen, R. E. Peale, C. J. Fredricksen, M. Ishigami, Univ. of Central Florida (United States); J. W. Cleary, Air Force Research Lab. (United States)

$90832 R \quad$ Characterization of an acoustic actuation mechanism for robotic propulsion in low Reynolds number environments [9083-103]

C. House, J. Armstrong, J. Burkhardt, S. Firebaugh, U.S. Naval Academy (United States)

$908325 \quad$ Nanoimprint-assisted directed self-assembly of low-molecular weight block copolymers: a route for 3D and multilevel nanostructures [9083-104]

C. Simao, W. Khunsin, N. Kehagias, A. Francone, Institut Català de Nanotecnologia (Spain); M. Zelsmann, Lab. des Technologies de la Microelectronique, CNRS (France);

M. A. Morris, Univ. College Cork (Ireland) and Trinity College Dublin (Ireland);

C. M. Sotomayor Torres, Institut Català de Nanotecnologia (Spain) and Institució Catalana de Recerca i Estudis Avançats (Spain)

9083 2T Modeling and simulation of multilayered thin films for terahertz detection [9083-107] M. F. Martin, U.S. Naval Academy (United States); F. Alves, Naval Postgraduate School (United States); R. A. T. Santos, Instituto Tecnológico de Aeronáutica (Brazil); D. Grbovic, Naval Postgraduate School (United States)

$90832 \mathrm{U} \quad$ Novel remote sensor systems: design, prototyping, and characterization [9083-108] V. Kayastha, S. Gibbons, J. E. Lamb III, Brewer Science, Inc. (United States); R. E. Giedd, Missouri State Univ. (United States) 
9083 2W Novel graphene FETs with field-controlling electrodes to improve RF performance [9083-1 10]

C. Al-Amin, R. Sinha, N. Pala, Florida International Univ. (United States); W. Choi, Univ. of North Texas (United States)

$90832 X \quad$ An optically resonant position read-out system for MEMS gas sensors [9083-1 12]

G. Putrino, A. Keating, M. Martyniuk, L. Faraone, J. M. Dell, The Univ. of Western Australia (Australia)

$90832 Z \quad$ Multiplexed optical operation of nanoelectromechanical systems (NEMS) arrays for sensing and signal-processing applications [9083-115]

A. Sampathkumar, Riverside Research Institute (United States)

908331 Imaging quality comparison of two typical methods for imaging through turbid media [9083-105]

X. Shao, T. Wu, C. Gong, Xidian Univ. (China)

908332 Sensing systems using chip-based spectrometers [9083-106]

A. Nitkowski, K. J. Preston, N. Sherwood-Droz, B. B. Behr, Tornado Spectral Systems (United States); Y. Bismilla, A. T. Cenko, B. DesRoches, J. T. Meade, E. A. Munro, J. Slaa,

B. S. Schmidt, A. R. Hajian, Tornado Spectral Systems (Canada)

908333 Graphene shield-enhancement of photosensitive surfaces and devices [9083-111] N. A. Moody, H. Yamaguchi, G. Gupta, A. D. Mohite, Los Alamos National Lab. (United States)

Author Index 


\title{
Conference Committee
}

\author{
Symposium Chair
}

David A. Whelan, Boeing Defense, Space, and Security (United States)

Symposium Co-chair

Nils R. Sandell Jr., Strategic Technology Office, DARPA (United States)

Conference Chairs

Thomas George, Zyomed Corporation (United States)

M. Saif Islam, University of California, Davis (United States)

Achyut K. Dutta, Banpil Photonics, Inc. (United States)

Conference Program Committee

Roger Appleby, Queen's University Belfast (United Kingdom)

Debjyoti Banerjee, Texas A\&M University (United States)

Scott D. Collins, University of Maine (United States)

Richard Conroy, National Institutes of Health (United States)

Nibir K. Dhar, Defense Advanced Research Projects Agency (United States)

Muhammad M. Hussain, King Abdullah University of Science and Technology (Saudi Arabia)

Matthew E. L. Jungwirth, Honeywell Defense and Space Electronic Systems (United States)

Christopher M. Kroninger, U.S. Army Research Laboratory (United States)

Susan M. Maley, U.S. Department of Energy (United States)

Michael C. McAlpine, Princeton University (United States)

Parvaneh Mokarian-Tabari, University College Cork (Ireland)

William D. Nothwang, U.S. Army Research Laboratory (United States)

Stergios J. Papadakis, Johns Hopkins University Applied Physics Laboratory (United States)

Michael K. Rafailov, University of Alberta (Canada)

Bilge Saruhan-Brings, Deutsches Zentrum für Luft- und Raumfahrt (Germany)

Antonio Sastre, National Institutes of Health (United States)

Noriko Satake, University of California, Davis Medical Center (United States)

Sivalingam Sivananthan, University of Illinois at Chicago (United States)

Andre U. Sokolnikov, Visual Solutions and Applications (United States)

Kyung-Ah Son, HRL Laboratories, LLC (United States)

Thomas G. Thundat, University of Alberta (Canada)

Christopher C. Wilcox, U.S. Naval Research Laboratory (United States) 
Session Chairs

1 Two-dimensional Nano-layered Systems: Graphene and Beyond

Thomas George, Zyomed Corporation (United States)

2 Emerging Electronic Devices/Systems Based on Adaptive and Metamaterials I

Andre U. Sokolnikov, Visual Solutions and Applications (United States)

3 Emerging Electronic Devices/Systems Based on Adaptive and Metamaterials II

Andre U. Sokolnikov, Visual Solutions and Applications (United States)

$4 \quad$ Graphene and 2D Electronics

Kyung-Ah Son, HRL Laboratories, LLC (United States)

5 ID Nanoelectronics: Nanowire and Nanotube Architecture Transistors

Muhammad M. Hussain, King Abdullah University of Science and Technology (Saudi Arabia)

6 MicroNano Technologies for Adaptive Optics and Beam Control

Christopher C. Wilcox, U.S. Naval Research Laboratory (United States)

Matthew E. Jungwirth, Honeywell Defense and Space Electronic Systems (United States)

7 MicroNano Sensor Systems for Power and Chemical Production Applications

Susan M. Maley, U.S. Department of Energy (United States)

Bilge Saruhan-Brings, Deutsches Zentrum für Luft- und Raumfahrt e.V. (Germany)

8 Advanced Micro/Nanotechnologies for Solar Energy Generation: Joint session with Conferences 9083 and 9115

Sivalingam Sivananthan, University of Illinois at Chicago (United States)

9 Mesodynamic Architectures

Ryan P. Lu, Federal Laboratory Consortium for Technology Transfer (United States)

10 3D Printing: An Emerging Technology for Micro/Nano Device Fabrication

Michael C. McAlpine, Princeton University (United States)

11 Flexible Electronics: Multifaceted Evolutions and Applications

Muhammad M. Hussain, King Abdullah University of Science and Technology (Saudi Arabia) 
12 Micro Autonomous Systems Technology (MAST): Performance Bounds and Trade Space Studies: Joint Session with Conferences 9083/9084/9096 Christopher M. Kroninger, U.S. Army Research Laboratory (United States) William D. Nothwang, U.S. Army Research Laboratory (United States)

13 Micro Autonomous Systems Technology (MAST): Power Solutions: Joint Session with Conferences 9083/9084/9096

William D. Nothwang, U.S. Army Research Laboratory (United States) Christopher M. Kroninger, U.S. Army Research Laboratory (United States)

14 Self-assembled, Block-copolymer, Nano-structures for Energy and Sensor Applications

Parvaneh Mokarian-Tabari, University College Cork (Ireland)

Michael A. Morris, University College Cork (Ireland)

15 Innovations in Multimodal Molecular Probes: Joint Session with Conferences 9083 and 9107

Antonio Sastre, National Institutes of Health (United States)

Richard Conroy, National Institutes of Health (United States)

16 High-accuracy Space-based Radiometry

Stergios J. Papadakis, Johns Hopkins University Applied Physics Laboratory (United States)

17 Nanotechnology for Millimeter-Wave Sensing I: Joint Session with Conferences 9083 and 9078

Michael K. Rafailov, University of Alberta (Canada)

18 Nanotechnology for Millimeter-Wave Sensing II: Joint Session with Conferences 9083 and 9078

Michael K. Rafailov, University of Alberta (Canada)

19 Micro/Nanotechnologies for Lasers and Standoff Detection I: Joint Session with Conferences 9083/9073/9106

Michael K. Rafailov, University of Alberta (Canada)

20 Micro/Nanotechnologies for Lasers and Standoff Detection II: Joint Session with Conferences 9083/9073/9106

Michael K. Rafailov, University of Alberta (Canada) 